Part of Journal of Research of the National Bureau of Standards, Volume 29, July 1942

\title{
EQUATION OF MOTION FOR THE STEADY MEAN FLOW OF WATER IN OPEN CHANNELS
}

\author{
By Garbis H. Keulegan
}

\section{ABSTRACT}

There is disagreement in the literature regarding the use of the Coriolis and the Boussinesq velocity-distribution coefficients in the open-channel flow equation. It is shown in this paper that the use of either coefficient is correct, provided the terms representing the effects of resistance are properly interpreted. The methods of deriving the two forms of the flow equation are given in detail, and it is shown that in the form of equation containing the Boussinesq coefficient, the friction coefficient is related directly to the wall friction. In the form of equation containing the Coriolis coefficient, the friction coefficient is related to the rate of energy loss in the water. This has a direct bearing on the correct use of Manning's " $n$ " in the equation of flow in open channels.

\section{CONTENTS}

I. Introduction _. 97

II. Purpose of paper

III. Reynolds equations of turbulent motion

IV. Energy principle in turbulent flow

V. Momentum equations for a stream tube

VI. Pressure law in gradually varied flow

VII. The two equations of open-channel flow

VIII. The coefficients of loss and friction in the varied flow equations__._._ 109

IX. Selecting the more useful form of the equation of motion for an open channel.

X. References

\section{INTRODUCTION}

The equation of motion of steady mean flow in open channels is the equation which describes, for the permanent regime of flow, the relation between the depth of water, the variation of this depth with distance along the bed of the channel, the mean velocity in a section, the variation of this velocity with distance, the slope of the bed, and the coefficient of resistance. The physical significance of the coefficient of resistance is determined by the manner in which the equation is derived. Essentially there are two methods of derivation, one of which involves the application of the energy equation, the other of a momentum equation, both of which result from the general hydrodynamic equations of motion. In addition to the quantities mentioned, the equation of motion contains a coefficient resulting from the velocity distribution in a section. The meaning of the latter, like that of the coefficient of resistance, depends on the way in which the equation is derived.

The results obtained by the two methods differ slightly. It seems that the significance of this difference and its bearing on the meaning of the coefficient of resistance are not generally recognized. Conse- 
quently, for investigations in the field of open-channel flow, the reasons for selecting one relation in preference to the other do not appear to be clearly understood today. Preference for one or the other relation depends, obviously, on the concept of the coefficient of friction which is adopted. It will be shown later in this paper that if the equation of motion is derived by the energy method, the concept underlying the friction coefficient in that equation is that of energy dissipation in the fluid per unit length of channel. On the other hand, if the momentum method is used, the concept of the friction coefficient is based on the frictional force exerted on the fluid by the walls of the channel.

\section{PURPOSE OF PAPER}

The purpose of this paper is to show the difference between the equation of steady mean flow in an open channel when derived by the energy method and the corresponding equation derived by the momentum method, and to explain the significance of this difference. Finally, it will be shown that the equation based on momentum considerations is the convenient one to use in discussing steady mean flow in open channels. Since the historical aspects of the problem have recently been discussed adequately by Bakhmeteff [1] ${ }^{1}$, they will not be given here.

\section{REYNOLDS EQUATIONS OF TURBULENT MOTION}

Before proceeding with the method of energy or momentum, it is necessary to adopt a system of dynamical equations of motion applicable to a liquid medium at the point $(x, y, z)$. The selection of these dynamical equations depends on whether the stresses due to relative motions are essentially viscous, or essentially apparent (turbulent), or in part viscous and in part apparent. Since the matter to be studied relates to turbulent flow in open channels, we may assume that in these channels the turbulent core occupies the whole cross section when the solid surfaces are covered with asperities; and the very small area of the laminar sublayer may be disregarded when the solid surfaces are smooth. We assume that the apparent stresses predominate everywhere in this core and that, therefore, no sensible error is introduced when the viscous stresses are neglected. The restrictions involved in these assumptions are not important for the general argument that will be followed in this paper. Neglecting the viscous stresses and denoting the temporal mean velocities in the directions of $x, y$, and $z$ by $u, v$, and $w$, respectively, the dynamical equations of motion to be adopted are of the form, assuming the liquid to be incompressible [2],

$$
\left.\begin{array}{l}
\rho \frac{d u}{d t}=-\frac{\partial p}{\partial x}-\rho \frac{\partial \Omega}{\partial x}+\frac{\partial R_{x x}}{\partial x}+\frac{\partial R_{y x}}{\partial y}+\frac{\partial R_{z x}}{\partial z} \\
\rho \frac{d v}{d t}=-\frac{\partial p}{\partial y}-\rho \frac{\partial \Omega}{\partial y}+\frac{\partial R_{x y}}{\partial x}+\frac{\partial R_{y y}}{\partial y}+\frac{\partial R_{z y}}{\partial z} \\
\rho \frac{d w}{d t}=-\frac{\partial p}{\partial z}-\rho \frac{\partial \Omega}{\partial z}+\frac{\partial R_{x z}}{\partial x}+\frac{\partial R_{y z}}{\partial y}+\frac{\partial R_{z z}}{\partial z}
\end{array}\right\}
$$

\footnotetext{
1 Figures in brackets indicate literature references at the end of this paper. Additional unnumbered references are given but not cited in the text.
} 
where $\Omega$ denotes the potential energy per unit mass at the point $(x, y, z)$, due to gravitational force; $\rho$ the density of the liquid; $p$ the temporal average of the pressure, and $R$ the apparent, or Reynolds, stresses. In terms of the velocity fluctuations, $u^{\prime}, v^{\prime}, w^{\prime}$, these latter stresses are given by

$$
\left.\begin{array}{ll}
R_{x x}=-\rho \overline{u^{\prime} u^{\prime}}, & R_{x y}=R_{y x}=-\overline{\rho u^{\prime} v^{\prime}} \\
R_{y y}=-\rho \overline{v^{\prime} v^{\prime}}, & R_{x z}=R_{z x}=-\rho \overline{u^{\prime} w^{\prime}} \\
R_{z z}=-\rho \overline{w^{\prime} w^{\prime}}, & R_{y z}=R_{z y}=-\rho \overline{v^{\prime} w^{\prime}}
\end{array}\right\}
$$

Since the flow is permanent, the operator $d / d t$ is

$$
\frac{d}{d t}=u \frac{\partial}{\partial x}+v \frac{\partial}{\partial y}+w \frac{\partial}{\partial z} \text {. }
$$

The effect of introducing the Reynolds stresses in the above equations is to give the mean temporal velocities, $u, v, w$, the character of velocities in stream-line flow. But the question then arises as to what we mean by a stream line or a fluid filament in turbulent flow. We shall define a fluid filament as a line for which the average directions of the instantaneous velocities at all points of the line are tangential to the line, or rather as a tubular surface composed of such lines and having a normal section of infinitely small dimensions. Stream lines are fixed in space, but, owing to turbulence, a particle of fluid initially on a stream line does not always remain on this line. However, if a surface made up of fluid filaments is selected, then the temporal average of the flow across this surface vanishes. For such a surface, if $u_{0}, v_{0}$, and $w_{0}$ are the velocity components of the mean motion at a point on the surface, and $l, m$, and $n$ are the direction cosines of a normal drawn to the surface,

$$
l u_{0}+m v_{0}+n w_{0}=0 .
$$

In turbulent flow the mean temporal velocities are subject to the compressibility condition, and this fact is denoted by the equation of continuity,

$$
\partial u / \partial x+\partial v / \partial y+\partial w / \partial z=0,
$$

which will be used frequently in this paper.

\section{ENERGY PRINCIPLE IN TURBULENT FLOW}

If we multiply the three dynamical equations, eq 1 , by $u, v, w$, respectively, use eq 3 , and add, we obtain, in view of the condition of compressibility, eq 5 ,

$$
\begin{gathered}
\frac{\rho}{2}\left[\frac{\partial}{\partial x}\left(u q^{2}\right)+\frac{\partial}{\partial y}\left(v q^{2}\right)+\frac{\partial}{\partial z}\left(w q^{2}\right)\right]=-\rho\left[\frac{\partial}{\partial x}(u \Omega)+\frac{\partial}{\partial y}(v \Omega)+\frac{\partial}{\partial z}(w \Omega)\right] \\
-\left[\frac{\partial}{\partial x}(u p)+\frac{\partial}{\partial y}(v p)+\frac{\partial}{\partial z}(w p)\right]+\frac{\partial}{\partial x}\left(u R_{x x}+v R_{x y}+w R_{x z}\right) \\
+\frac{\partial}{\partial y}\left(u R_{y x}+v R_{y y}+w R_{y z}\right)+\frac{\partial}{\partial z}\left(u R_{z x}+v R_{z y}+w R_{z z}\right)-\Psi
\end{gathered}
$$


where $q$ is the absolute velocity,

$$
q^{2}=u^{2}+v^{2}+w^{2},
$$

and $\Psi$ is the turbulent dissipation function:

$$
\begin{aligned}
\Psi=R_{x x} \frac{\partial u}{\partial x}+R_{y y} \frac{\partial v}{\partial y}+ & R_{z z} \frac{\partial w}{\partial z}+R_{x y}\left(\frac{\partial u}{\partial y}+\frac{\partial v}{\partial x}\right)+R_{x z}\left(\frac{\partial u}{\partial z}+\frac{\partial w}{\partial x}\right) \\
& +R_{y z}\left(\frac{\partial v}{\partial z}+\frac{\partial w}{\partial y}\right) .
\end{aligned}
$$

The physical meaning of eq 6 can be explained in a concise manner, if we multiply the two sides of eq 6 by $d x d y d z$, perform the integration for a volume $V$, such as shown in figure 1 , and consider the result.

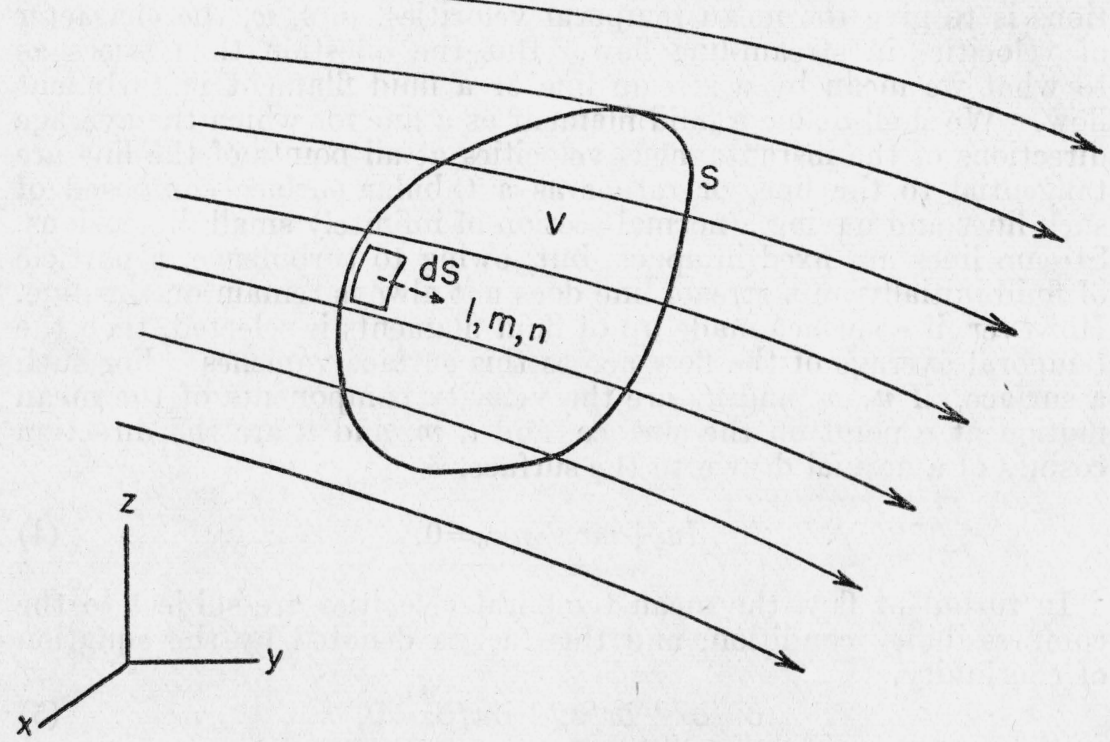

Figure 1.-A closed surface in a field of flow.

To facilitate the required integration use may be made of Green's theorem in three dimensions (also referred to as Gauss' theorem or the divergence theorem),

$$
\int_{V}\left(\frac{\partial X}{\partial x}+\frac{\partial Y}{\partial y}+\frac{\partial Z}{\partial z}\right) d V=-\int_{S}(l X+m Y+n Z) d S
$$

where $X, Y, Z$ are any three functions, finite, single valued, and differentiable at all points of the connected region $V$ completely bounded by one or more closed surfaces, $S$. The quantities $l, m, n$ are the direction cosines of the normal drawn inwards. An element of volume is denoted by $d V$, and an element of surface by $d S$. The volume 
integral is taken throughout the region of $V$ and the surface integral on the boundaries $S$. Effecting the integration, we find

where

$$
\begin{gathered}
\int_{S}\left(p+\frac{\rho}{2} q^{2}+\rho \Omega\right)(l u+m v+n w) d S= \\
\int_{S}\left(u R_{h x}+v R_{h y}+w R_{h z}\right) d S+\int_{V} \Psi d V,
\end{gathered}
$$

$$
\left.\begin{array}{l}
R_{h x}=l R_{x x}+m R_{y x}+n R_{z x} \\
R_{h y}=l R_{x y}+m R_{y y}+n R_{z y} \\
R_{h z}=l R_{x z}+m R_{y z}+n R_{z z}
\end{array}\right\}
$$

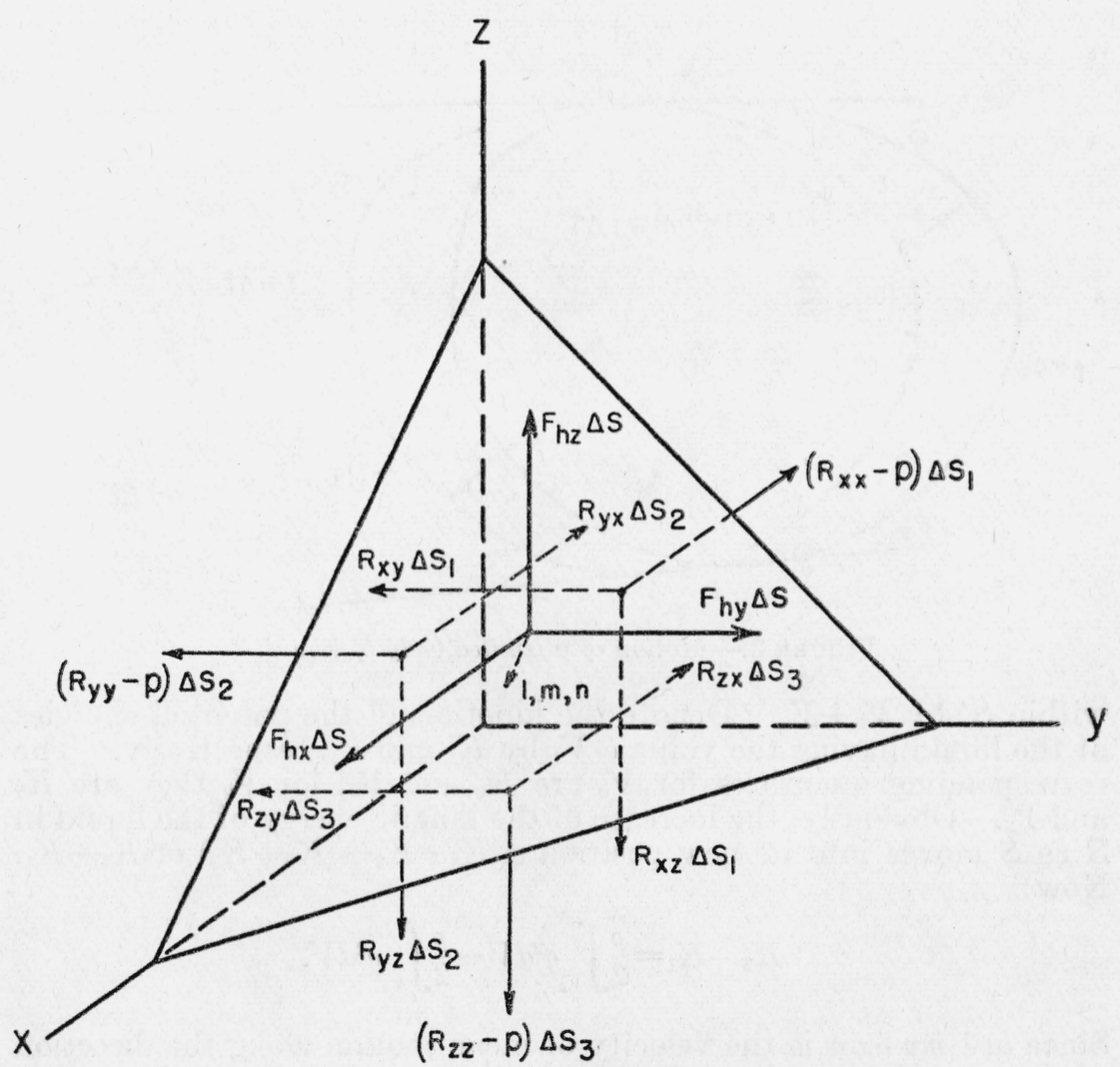

Figure 2.-Boundary stresses expressed in terms of Reynolds stresses by means of tetrahedron.

To give physical significance to $R_{h x}, R_{h y}$, and $R_{h z}$, consider the force $F d S$ which the liquid outside of $S$ exerts on $d S, d S$ being on the boundary of $V$. See figure 2. The components of $F d S$ in the three directions of $x, y, z$ are $F_{h x} d S, F_{h y} d S, F_{h z} d s$, respectively. Using Cauchy's theorem of the tetrahedron, we find, in view of eq 11, the expressions 


$$
\left.\begin{array}{l}
-F_{h x}=R_{h x}-l p, \\
-F_{h y}=R_{h y}-m p \\
-F_{h z}=R_{h z}-n p .
\end{array}\right\}
$$

Hence $R_{h x}, R_{h y}, R_{h z}$ are the boundary stresses on the liquid outside of $V$ with the pressures removed. They are the boundary stresses due to turbulence.

To interpret the relation in eq 10 consider figure 3 . In this figure, $S$ and $S^{\prime}$ represent the envelopes of the same body of liquid at the times $t$ and $t+\Delta t$, respectively, in the sense of stream-line flow. Denote the volume of liquid within the surface $S$ by $V_{1}+V_{0}$; that

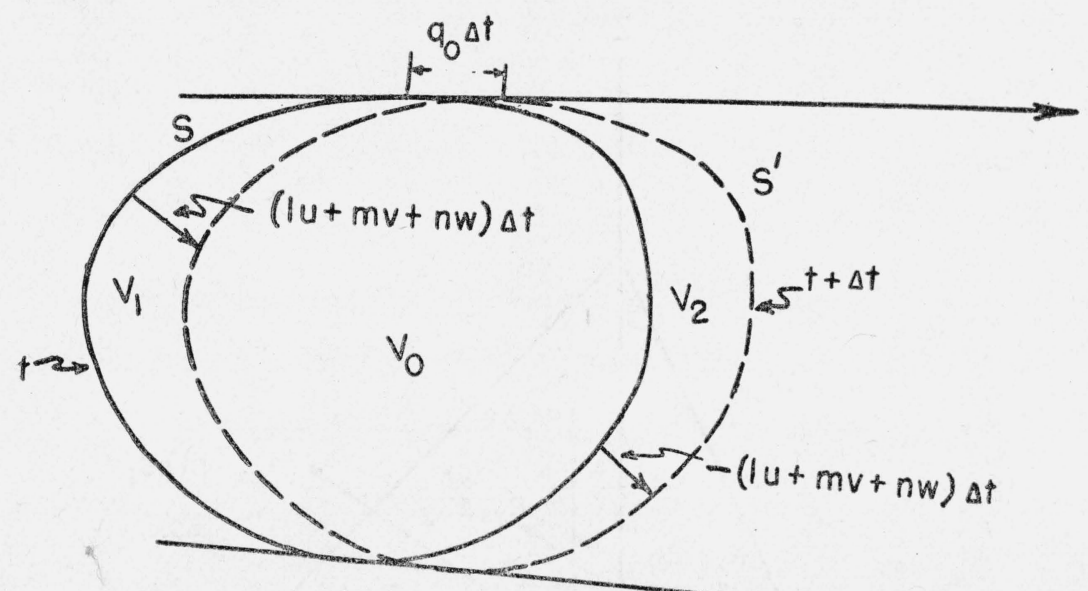

Fradre 3.-Motion of a limited body of liquid.

within $S^{\prime}$ by $V_{2}+V_{0}$. Denote the kinetic and the potential energies of the liquid having the volume $V_{1}$ by $K_{1}$ and $\mathrm{P}_{1}$, respectively. The corresponding quantities for $V_{0}$ are $K_{0}$ and $\mathrm{P}_{0}$; for $V_{2}$ they are $K_{2}$ and $\mathrm{P}_{2}$. Obviously, the increase of the kinetic energy of the liquid in $S$ as $S$ moves into its new position is $K_{0}+K_{2}-\left(K_{0}+K_{1}\right)$ or $K_{2}-K_{1}$. Now

$$
K_{2}-K_{1}=\frac{\rho}{2} \int_{V_{2}} q^{2} d V-\frac{\rho}{2} \int_{V_{1}} q^{2} d V .
$$

Since $l u+m v+n w$ is the velocity of mean motion along the direction of the normal drawn inwards,

$$
\begin{aligned}
& \text { for } V_{1}: d V=(l u+m v+n w) d S \Delta t, \\
& \text { for } V_{2}: d V=-(l u+m v+n w) d S \Delta t,
\end{aligned}
$$

and hence

$$
K_{2}-K_{1}=-\Delta t \frac{\rho}{2} \int_{S} q^{2}(l u+m v+n w) d S
$$


It can be shown in a similar manner that the increase of potential energy is

$$
P_{2}-P_{1}=-\Delta t \rho \int_{s} \Omega(l u+m v+n w) d S .
$$

On the other hand, the work done by the pressure, $p$, on the liquid in $S$ at time $t$ during the subsequent interval $\Delta t$ is

$$
W=\Delta t \int_{s} p(l u+m v+n w) d S .
$$

Accordingly, the terms on the left-hand side of eq 10 give the excess of the work done on the liquid by pressure in that portion of the liquid bounded by the surface $S$ over the variation of the kinetic and potential energies of the same liquid, all per unit time. The kinetic energy of the liquid in this case is the energy of mean motion. The right-hand member gives the dissipation in the body of water inside of the surface $S$ due to the Reynolds stresses and the work done by these stresses on the liquid outside of $S$. In other words, the equation states:

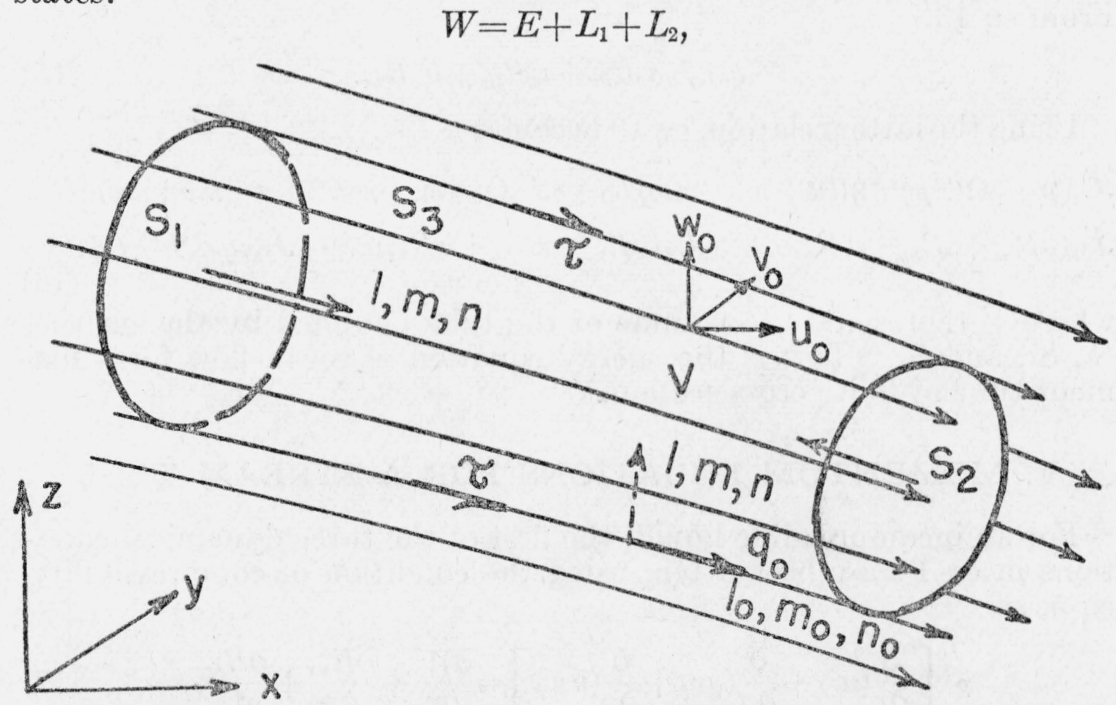

FIgURe 4.- Segment of a tube of fluid filaments.

where $W$ is the work done on the fluid in $S$ by the external hydrostatic pressure, $E$ is the increase in energy, $L_{1}$ is the work done by the fluid inside of $S$ against turbulent boundary stresses, and $L_{2}$ is the energy dissipated.

Obviously eq 10 is the energy equation for steady mean motion. The consideration of the equation becomes more fruitful if we select for the volume $V$ the region bounded by a tube of fluid filaments and two planes, the planes forming the two ends of the tube. See figure 4. Let $S_{3}$ represent the surface formed by the outer fluid filaments; $S_{1}$ the surface at the end of the tube through which the liquid is entering; and $S_{2}$ the surface at the other end of the tube, through which the liquid is leaving. There is no flow across the bounding surface; that is, the mean motion across it is zero; hence the mean motion at $S_{3}$ is tangen- 
tial. Let $q_{0}$ denote the absolute velocity of flow at this surface and let $u_{0}, v_{0}, w_{0}$ be its components. Denote the direction cosines of $q_{0}$ by $l_{0}, m_{0}, n_{0}$.

Hence

$$
u_{0}=q_{0} l_{0}, v_{0}=q_{0} m_{0}, w_{0}=q_{0} n_{0} ;
$$

and, in view of eq 4 ,

$$
l l_{0}+m m_{0}+n n_{0}=0 .
$$

Next, consider the surface force $+F d S$ exerted on $d S$, an element of $S_{3}$, by the liquid outside the tube, and denote its component in the direction of $q_{0}$ by $\tau d S$. Then

$$
\tau=l_{0} F_{h x}+m_{0} F_{h y}+n_{0} F_{h x} \text {. }
$$

From eq 12 and 14

$$
-\tau=l_{0} R_{h x}+m_{0} R_{h y}+n_{0} R_{h z} .
$$

From eq 13

$$
-q_{0} \tau=u_{0} R_{h x}+v_{0} R_{h y}+w_{0} R_{h z} .
$$

Using the latter relation, eq 10 becomes

$\int_{s_{1}}\left(p+\rho \Omega+\rho q^{2} / 2\right)(l u+m v+n w) d S+\rho \int_{s_{2}}\left(p+\rho \Omega+\rho q^{2} / 2\right)(l u+m v+n w) d S=$ $\int_{s_{1}}\left(u R_{h x}+v R_{h y}+w R_{h z}\right) d S+\int_{s_{2}}\left(u R_{h x}+v R_{h y}+w R_{h z}\right) d S-\mathcal{S}_{s_{3}} \tau q_{0} d S+\mathcal{S}_{V} \Psi d \mathrm{~V}$,

where $V$ represents the volume of the tube bounded by the surfaces $S_{1}, S_{2}$, and $S_{3}$. This is the energy equation of mean flow for a filament tube of finite cross section.

\section{MOMENTUM EQUATIONS FOR A STREAM TUBE}

For an incompressible liquid, the first of the three dynamical equations in eq 1 may be written, using the condition of compressibility, eq 5 , as

$$
\rho\left[\frac{\partial}{\partial x}\left(u^{2}\right)+\frac{\partial}{\partial y}(u v)+\frac{\partial}{\partial z}(u w)\right]=\frac{\partial \Pi_{x}}{\partial x}+\frac{\partial R_{v x}}{\partial y}+\frac{\partial R_{z x}}{\partial z},
$$

where

$$
\Pi_{x}=-p-\rho \Omega+R_{x x} .
$$

Multiplying the two members of this by $d x d y d z$ or $d V$, integrating through the space $V$ of the tube in figure 4, and again using Green's theorem with inward-drawn normal, we obtain, after changing the signs:

$$
\begin{array}{r}
\rho \mathcal{S}_{s_{1}} u(l u+m v+n w) d S+\mathcal{S}_{s_{2}} u(l u+m v+n w) d S=\mathcal{J}_{s_{1}}\left(l \Pi_{x}+m R_{y x}+n R_{x z}\right) d S \\
+\int_{s_{2}}\left(l \Pi_{x}+m R_{y x}+n R_{x z}\right) d S+\mathcal{S}_{s_{3}}\left(l \Pi_{x}+m R_{y x}+n R_{x z}\right) d S .
\end{array}
$$

The integral on the left over $S_{3}$ vanishes, since, by eq 13 and 14 ,

$$
l_{0} u_{0}+m_{0} v_{0}+n_{0} w_{0}=0 \text {. }
$$


This is the momentum equation for the flow through the tube with reference to the $x$-direction, which states that the time rate of change of the momentum equals the $x$-component of the forces acting. The equation suggests that, to evaluate the difference of momenta of the liquid entering through $S_{1}$ and leaving through $S_{2}$, it is necessary to know the values of the pressure, the Reynolds stresses, and the potential in respect to the gravitational force at the bounding surfaces, and the direction cosines of the normals to these surfaces. The momentum equations corresponding to the $y$ and $z$ directions may be derived in a similar manner. A simpler method would be to obtain them from eq 19 by applying the cyclic permutations $u \rightarrow v \rightarrow w, x \rightarrow y \rightarrow z$, and $l \rightarrow m \rightarrow n$.

\section{PRESSURE LAW IN GRADUALLY VARIED FLOW}

The application of the energy and momentum equations to openchannel flow will give practical results only when some definite restrictions are imposed on the form of channel and on the variation of the depth of water. Otherwise, too many quantities difficult to evaluate numerically will appear in the results.

Taking the $x$-axis in the bed of the channel, the positive branch being in the direction of flow, the $y$-axis horizontal, positive $z$-axis directed upwards, the equation of the surface forming the channel may be given by

$$
z_{1}=f\left(x_{1}, y_{1}\right),
$$

where $x_{1}, y_{1}, z_{1}$, are the coordinates of a point on the channel boundary. Denote the direction cosines of the inward-drawn normal to this surface by $l_{1}, m_{1}, n_{1}$. The restriction of the channel form to be assumed is that $l_{1}$ and $H \partial l_{1} / \partial x$, where $H$ is depth of water, are small quantities, the squares of these being negligible with respect to unity. We take as the depth of water the $z$-coordinate of the free surface. The restriction of the depth of water to be assumed is that, $H$ being a function of $x$ only, $H d^{2} H / d x^{2}$ and the square of $d H / d x$ are negligible with respect to unity. In Boussinesq's language, the flow is "gradually varied". These restrictions isolate the localities where the slope of the bed or the cross section of the channel changes abruptly. They isolate also the places where hydraulic jumps or bends of the watercourse occur.

The restrictions made in the above have the following kinematical and dynamical consequences. The squares, $v^{2}$ and $w^{2}$, are negligible with respect to $u^{2}$, and hence $q^{2}=u^{2}$. The derivatives of the ratios $w / u$ and $v / u$ with respect to $x$ are negligible in comparison with the derivatives of these ratios with respect to $y$ and $z$. Then the accelerations in the directions of $y$ and $z$ are negligible; that is, the lefthand terms in the second and the third equations in eq 1 may be discarded [3].

In addition to the above, it becomes necessary to make certain assumptions in regard to the Reynolds stresses. First, the velocity fluctuations are such that

$$
\overline{u^{\prime} u^{\prime}}=\overline{v^{\prime} v^{\prime}}=\overline{w^{\prime} w^{\prime}} \text {; }
$$

that is, $R_{x x}=R_{y y}=R_{z z}$, and thus $\Pi_{x}=\Pi_{y}=\Pi_{z}=\Pi$. 
Second, $u$ being the predominant component, the correlation between the fluctuations $v^{\prime}$ and $w^{\prime}$ and the derivatives of this correlation with respect to $y$ and $z$ are negligible in comparison with the correlation between $u^{\prime}, v^{\prime}$ and $v^{\prime}, w^{\prime}$ and the corresponding derivatives. That is, the derivatives of $R_{z y}$ with respect to $y$ and $z$ may be neglected. Third, since the flow is gradually varied, the $x$-derivatives of $R_{x y}$ and $R_{x z}$ may be ignored.

With these assumptions in mind, the second and the third equations in eq 1 reduce to

and

$$
\frac{\partial p}{\partial y}+\rho \frac{\partial \Omega}{\partial y}-\frac{\partial R_{y y}}{\partial y}=0
$$

$$
\frac{\partial p}{\partial z}+\rho \frac{\partial \Omega}{\partial z}-\frac{\partial R_{z z}}{\partial z}=0
$$

Take the horizontal plane passing through the point $P(0,0,0)$ as the surface of reference for the potential energy. Denote the bed inclination, that is, the angle between the $x$-axis and this plane by $i$. Then

$$
\rho \Omega=\rho g z-i x g \rho
$$

assuming that $i$ is small. The general solution of eq 21 is

$$
p+\rho g z-i x g \rho-R_{z z}=f(x, y),
$$

where $f$ is an arbitrary function. At the free surface, that is, at $z+H$, the pressure is atmospheric, so that $p=p_{a}$. Obviously, the fluctuations $w^{\prime}$ vanish at the free surface, or $R_{z z}=0$ at $z=H$. Hence

$$
p_{a}+\rho g H-\rho i x g=f(x, y) \text {. }
$$

Subtracting, we have

$$
p=\rho g(H-z)+R_{z z}+p_{a},
$$

or, since $R_{x x}=R_{z z}=R_{y y}$,

$$
p=\rho g(H-z)+R_{x x}+p_{a},
$$

which relation satisfies eq 20 also. This is the law of pressures in gradually varied flow. Differentiating eq 18, and using eq 22 and 23 , we obtain

$$
-\frac{\partial \Pi}{\partial x}=\rho g \frac{d H}{d x}-\rho g i
$$

\section{THE TWO EQUATIONS OF OPEN-CHANNEL FLOW}

Let us first consider the energy method. We regard the portion of water in the channel between the planes $x=x_{1}$ and $x=x_{2}$ as the filament tube for which the energy relation of eq 16 is to be evaluated. See figure 5 . The sections $A_{1}$ at $x_{1}$ and $A_{2}$ at $x_{2}$ are identified with $S_{1}$ and $S_{2}$, respectively. The free surface and the wetted surface between the sections at $x_{1}$ and $x_{2}$ constitute the surface $S_{3}$. Accordingly, $q_{0}$ is the velocity of water at the free surface or in the plane of the peaks of the asperities of the solid boundary, or the velocity at the face of the laminar sublayer if the bounding surface is smooth. At the section $A_{1}, l=1$, 
$m=0, n=0 . \quad$ At $A_{2}, l=-1, m=0$, and $n=0$. At the surface $S_{3}, l=l_{1}$, $m=m_{1}$, and $n=n_{1}$. It must be remembered that $l_{1}$ is small. The energy relation for the flow in channel from eq 16 is thus, using eq 11:

$$
\begin{aligned}
& \int_{A_{1}}\left(\frac{\rho u^{2}}{2}+\rho \Omega+p-R_{x x}\right) u d A-\int_{A_{2}}\left(\frac{u^{2}}{2}+\rho \Omega+p-R_{x x}\right) u d A= \\
& \int_{A_{1}}\left(v R_{x y}+w R_{x z}\right) d A-\int_{A_{2}}\left(v R_{x y}+w R_{x z}\right) d A-\int_{S_{3}} \tau q_{0} d S+\int_{V} \Psi d V .
\end{aligned}
$$

Since we have assumed that $R_{x x}=R_{y y}=R_{z z}$ and that the liquid is incompressible, the dissipation function $\Psi$ as given in eq 8 now takes on the form

$$
\Psi=R_{x y}\left(\frac{\partial u}{\partial y}+\frac{\partial v}{\partial x}\right)+R_{x z}\left(\frac{\partial u}{\partial z}+\frac{\partial w}{\partial x}\right)+R_{y 2}\left(\frac{\partial v}{\partial z}+\frac{\partial w}{\partial y}\right) .
$$

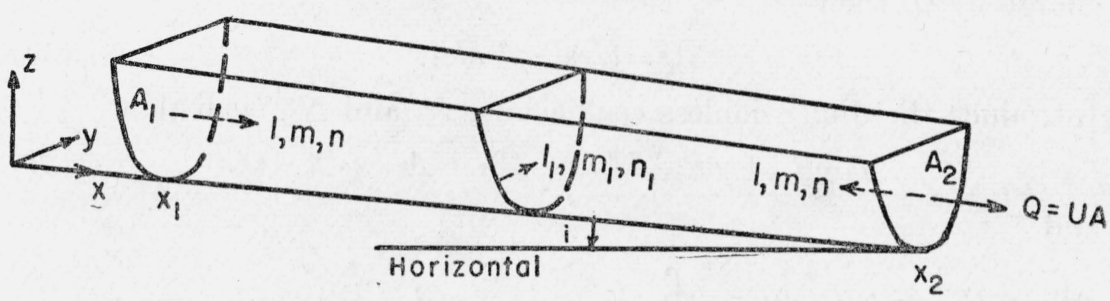

Figure 5.-Segment of a tube of fluid filaments in gradually varied flow.

Since we are dealing with gradually varied flow, the value of the product $\tau q_{0}$ in terms of the Reynolds stresses and the wall velocities takes on a simpler form. The general value of this product is, from eq 15 and 11 ,

$$
\begin{aligned}
-\tau q_{0}=u_{0}\left(l_{1} R_{x x}+m_{1} R_{y x}+n_{1} R_{z x}\right)+v_{0}\left(l_{1} R_{x y}+m_{1} R_{y y}+n_{1} R_{z y}\right)+ & w_{0}\left(l_{1} R_{x z}+m_{1} R_{y z}+n_{1} R_{z z}\right) .
\end{aligned}
$$

Using eq 4 and remembering that $R_{x x}=R_{y v}=R_{z z}$, this simplifies to

$$
-\tau q_{0}=\left(u_{0} m_{1}+v_{0} l_{1}\right) R_{y x}+\left(u_{0} n_{1}+w_{0} l_{1}\right) R_{z x}+\left(v_{0} n_{1}+w_{0} m_{1}\right) R_{y z} .
$$

Since the flow is gradually varied, $v_{\mathrm{n}} l_{1}$ is negligible in comparison with $u_{0} m_{1}$, and $w_{0} l_{1}$ in comparison with $u_{0} n_{1}$, and thus we have

$$
-\tau q_{0}=u_{0} m_{1} R_{y x}+u_{0} n_{1} R_{z x}+\left(v_{0} n_{1}+w_{0} m_{1}\right) R_{y 2} .
$$

We wish to obtain the channel equation in its differential form. To do this we select $x_{1}=x$ and $x_{2}=x+d x$. We suppose that the Reynolds stresses vanish at the free surface, and remember that $d V=A d x$, $n_{1} d S=d y_{1} d x_{1}$ and $m_{1} d S=-d z_{1} d x_{1}$, since we shall evaluate the line integrals counterclockwise in the $y z$-plane.

Then eq 25 becomes

$$
\begin{aligned}
& -\frac{d}{d x} \int_{A}\left(\rho u^{2} / 2+\rho \Omega+p-R_{x x}\right) u d A=-\frac{d}{d x} \int_{A}\left(v R_{x y}+w R_{x z}\right) d A- \\
& \oint_{P_{W}} u_{0}\left(R_{y x} d z_{1}-R_{z x} d y_{1}\right)+\oint_{P_{W}} R y z\left(v_{0} d y_{1}-w_{0} d z_{1}\right)+\int_{A} \Psi d A,
\end{aligned}
$$


where $A$ is the cross section at $x, P_{W}$ is the wetted perimeter, and $d z_{1}$ and $d y_{1}$ are the projections of an arc $d s$ of the wetted perimeter.

Next we express the left-hand terms of the last equation in terms of a quantity which is measurable when the channel is prismatic and the flow is uniform. The measurable quantity that will be used is

$$
\oint_{P_{W}} R_{y x} d z_{1}-R_{z x} d y_{1}=-\tau_{0} P_{W}
$$

where $\tau_{0}$ in the case of uniform flow in an open channel is the magnitude of the average shear on the wall per unit length of channel and is a positive quantity. Denote the hydraulic radius by $R$, so that

$$
R P_{W}=A \text {. }
$$

Denote the average velocity in the direction of $x$ by $U$ and the discharge by $Q$ : then

$$
Q=U A=\int_{4} u d A \text {. }
$$

Introduce the dimensionless coefficients, $N_{1}$ and $N_{2}$, such that

$$
\mathcal{S}_{A}\left(v R_{x y}+w R_{z x}\right) d A=-N_{1} \tau_{0} U A=-N_{1} \tau_{0} Q,
$$

and

$$
\oint_{P_{W}} u_{0}\left(R_{y x} d z_{1}-R_{z x} d y_{1}\right)-\oint_{P_{W}} R_{y z}\left(v_{0} d y_{1}-w_{0} d z_{1}\right)-\int_{A} \Psi d A=-N_{2} \tau_{0} Q / R
$$

In addition to these we introduce also the well-known dimensionless coefficient $\alpha$ of Coriolis; which is defined by

$$
\int_{A} u^{3} d A=\alpha U^{3} A=\alpha U^{2} Q .
$$

In view of eq $24,30,31,32$, and 33 , eq 28 now may be given in the form

$$
g \frac{d H}{d x}+\alpha U \frac{d U}{d x}=g i-\frac{d}{d x}\left(N_{1} \tau_{0} / \rho\right)-N_{2} \tau_{0} / \rho R
$$

which is the equation of motion of steady flow in open channels, as derived by the energy method.

Consider secondly the momentum method. Working with the same fluid tube as in the above we have, from eq 19, after transforming the $I I$ terms back into a volume integral, the relation,

$$
\int_{A_{1}} \rho u^{2} d A-\int_{A_{2}} \rho u^{2} d A=-\int_{V} \frac{\partial \Pi}{\partial x} d V+\int_{S_{3}}\left(m_{1} R_{y x}+n_{1} R_{z x}\right) d S .
$$

The differential relation derived from this, by putting $x_{1}=x$ and $x_{2}=x+d x, d V=A d x, n_{1} d S=d y_{1} d x_{1}, m_{1} d S=-d z_{1} d x_{1}$, reduces to

$$
-\frac{d}{d x} \int_{A} u^{2} d A=-\int_{A} \frac{\partial \Pi}{\partial x} d A+\oint_{P_{W}}\left(R_{z x} d y_{1}-R_{y x} d z_{1}\right) .
$$

Now introduce the Boussinesq coefficient $\eta$ by means of the relation

$$
(1+\eta) U^{2} A=\int_{A} u^{2} d A,
$$


or

$$
(1+\eta) U Q=\int_{A} u^{2} d A
$$

Differentiating both sides of this equation by $x$ and assuming that $\eta$ changes slowly with $x$, we have

$$
\frac{d}{d x} \int_{A} u^{2} d A=(1+\eta) Q \frac{d U}{d x}
$$

In view of eq $18,22,24$, and 37 , eq 35 , after dividing by $\rho A$, reduces to

$$
g \frac{d H}{d x}+(1+\eta) U \frac{d U}{d x}=g i-\tau_{0} / \rho R,
$$

which is the equation of motion of steady flow in open channels, as derived by the momentum method.

\section{THE COEFFICIENT OF LOSS AND FRICTION IN THE VARIED-FLOW EQUATIONS}

The two equations of motion, eq 34 and 38 , of varied flow in open channels given above, are in conformity with theory. Examining these two equations, it must be emphasized that the equation derived from the general energy equation, that is, eq 34, contains $N_{1}$ and $N_{2}$ in addition to $\tau_{0}$. All three of these quantities are unknown even if we suppose that $H, U$, and $\alpha$ are known as functions of $x$, through observation. Since by the mere observation of $H, U$, and $\alpha$, the quantities $\tau_{0}, N_{1}$, and $N_{2}$ can not be determined, the terms involving these unknowns may be represented as a single term. That is, we put

$$
R \frac{d}{d x}\left(N_{1} \tau_{0}\right)+N_{2} \tau_{0}=+\lambda_{e} \cdot \frac{1}{2} \rho U^{2},
$$

and then eq 34 takes the form

$$
g \frac{d H}{d x}+\alpha U \frac{d U}{d x}=g i-\lambda_{e} \frac{U^{2}}{2 R},
$$

where $\lambda_{e}$ is a dimensionless coefficient arising solely from the fact that there is dissipation of energy in the flow. The quantity

$$
\lambda_{e} \frac{U^{2}}{2 R} \cdot \rho Q \text {, }
$$

where $Q$ is the constant discharge in the open-channel flow, represents the rate of loss of energy in the channel per unit length of channel. This interpretation follows from the fact that the results were derived from the general energy equation. Accordingly, we must look upon $\lambda_{e}$ as a coefficient of energy loss.

On the other hand, the equation derived from the general momentum equation, that is, eq 38 , contains the single unknown, $\tau_{0}$. If we write

$$
\tau_{0}=\lambda \rho \frac{U^{2}}{2}
$$

eq 38 takes on the form

$$
g \frac{d H}{d x}+(1+\eta) U \frac{d U}{d x}=g i-\lambda \frac{U^{2}}{2 R}
$$


Since $\tau_{0}$ is the frictional force of the channel per unit length, the coefficient $\lambda$ represents the coefficient of friction.

\section{SELECTING THE MORE USEFUL FORM OF THE EQUA. TION OF MOTION FOR AN OPEN CHANNEL}

If the equations above given were equally advantageous, a selection between the two would be meaningless. But this is not the case. Of the coefficients, $\lambda_{e}$ and $\lambda$, the second being directly associated with the friction exerted by the walls of channel is the less affected by changes in $d U / d x$. This is obvious when we consider the equation which expresses the coefficients in terms of the shear. Judging solely on this basis, the universal use of eq 42 as the equation of motion for varied flow in open channels would naturally be favored.

In this connection an error that is often made in the use of the equations above discussed should be pointed out. Frequently, eq 40 is employed to determine Manning's " $n$ " for open channels. Since Manning's " $n$ " relates to the magnitude of the frictional force in channels, obviously the correct equation to use is eq 42 , the relation between $n$ and $\lambda$ being

$$
n=1.486 R^{1 / 3}(\lambda / 2 g)^{1 / 2},
$$

where $R$ and $g$ are measured in English units; that is, in feet and in seconds.

The following general remarks regarding the role of the energy and the momentum equations in hydrodynamics may be made in passing. In the present problem we are concerned solely with the relation between wall resistance and mean flow in the channel, and hence no advantage is achieved by the simultaneous use of the energy and momentum equations. The reason for this is that the law of velocity distribution in open channels, when the flow is turbulent, is not susceptible of theoretical determination. Experience establishes the relation between the local velocities and the shear at the wall. This being the case, one of these equations is sufficient, as explained above. But if the problem considered is one in streamline flow, and if solutions are attempted by approximate methods, then the simultaneous use of the energy and momentum equations results in improving the approximations of the solutions obtained.

In solving the problems of boundary layer on a plate and at the entrance section of a pipe, for example, it is necessary first to establish the law of velocity distribution. It has been customary to use the momentum relation to obtain the approximate form of this distribution. If, in addition, the energy relation is used, a better approximation results.

The author expresses his appreciation to George W. Patterson and Herbert N. Eaton for valuable criticism and review of the paper.

\section{REFERENCES}

[1] B. A. Bakhmeteff, Coriolis and the energy principle in hydraulics, Applied Mechanics, Theodore von Kármán Anniversary Volume, p. 59 (1941).

[2] Hunter Rouse, Fluid Mechanics for Hydraulic Engineers, p. 179 (McGrawHill Book Co., New York, N. Y., 1938).

[3] J. Boussinesq, Essai sur la théorie des eaux courantes, Mém. divers Savants Acad. Sci. 32, 56 (1891). 


\section{ADDITIONAL REFERENCES}

G. G. Coriolis. Sur l'établissement de la formule qui donne la figure des remous, et sur la correction qu'on doit y introluire pour tenir compte des différences de vitesse dans les divers points d'une mêr e section d'un courant. Annales des Ponts et Chaussées, 1st semester (1836) (se ies 1, vol. 11).

Vauthier, Note sur la correction que $M$. l'ingénieur en chef Coriolis propose de faire subir à la formule du mouvement permanent des eaux courantes, pour tenir compte des differences de vitesse des molecules fluides, aux divers points d'une même section transversale du courant. Annales des Ponts et Chaussées, $2 \mathrm{~d}$ semester (1836) (series 1, vol. 12).

A. J. C. B. de Saint-Venant, Des divers manières de poser les equations du mouvement varié des eaux courantes. Annales des Ponts et Chaussées, 1st semester (1887) (series 6, vol. 13).

Washington, May 15, 1942. 
NATIONAL BUREAU OF STANDARDS,

WASHINGTON, D. C.

Send me the Mathematical Tables marked X below. I enclose remittance ${ }^{1}$ to cover the cost.

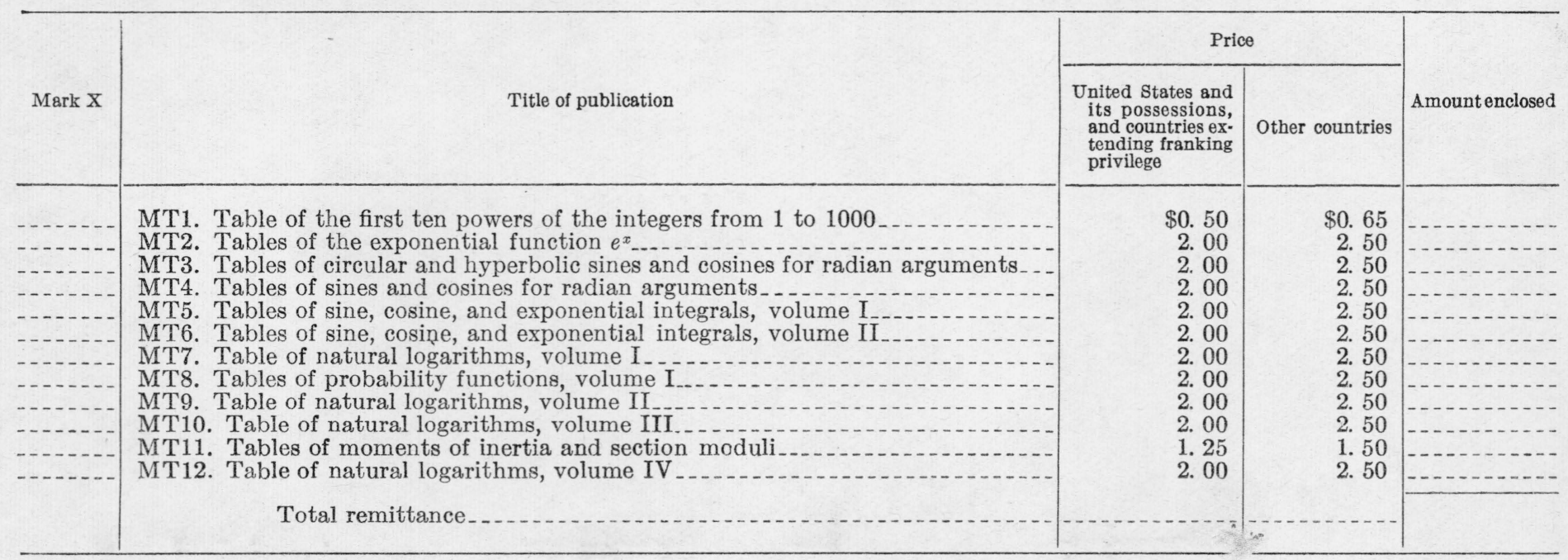

${ }^{1}$ Remittance should be in form of post-office money order, or check, and made payable to the order of the "National Bureau of Standards" in United States currency.

Send to

Number and Street

City and State 


\section{MATHEMATICAL TABLES}

Attention is invited to a series of publications which is being prepared by the Project for the Computation of Mathematical Tables conducted by the Federal Works Agency, Work Projects Administration for the City of New York under the sponsorship of the National Bureau of Standards.

To date, twelve tables have been made available through the National Bureau of Standards. These are listed below:

MT1. Table of the First Ten Powers of the Integers From 1 to 1000:

(1938) VIII+80 pages; heavy paper cover. 50 cents.

MT2. Tables of the Exponential Function $e^{x}$ :

The ranges and intervals of the argument and the number of decimal places in the entries are given below:

$\begin{array}{ccc}\text { Range of } x & \text { Interval of } x & \text { Decimals given } \\ -2.5000 \text { to } 1.0000 & 0.0001 & 18 \\ 1.0000 \text { to } 2.5000 & .0001 & 15 \\ 2.500 \text { to } 5.000 & .001 & 15 \\ 5.00 \text { to } 10.0 & .01 & 12\end{array}$

(1939) XV + 535 pages; bound in buckram, $\$ 2.00$.

MT3. Tables of Circular and Hyperbolic Sines and Cosines for Radian Arguments:

Contains 9 decimal place values of $\sin x, \cos x, \sinh x$ and $\cosh x$ for $x$ (in radians) ranging from 0 to 2 at intervals of 0.0001 .

(1939) XVII+405 pages; bound in buckram, $\$ 2.00$.

MT4. Ta bles of Sines and Cosines for Radian Arguments:

Contains 8 decimal place values of sines and a vines for radian arguments ranging from 0 to 25 at intervals of 0.001 .

(1940) XXIX + 275 pages; bound in buckram, $\$ 2.00$.

MT5. Tables of Sine, Cosine, and Exponentral Integrais, volumb I:

Values of these functions to 9 places of decimals from 0 to 2 at intervals of 0.0001 .

(1940) XXVI+444 pages; bound in buckram, $\$ 2.00$.

MT6. Tables of Sine, Cosine, and Exponential. Integrals, Volume II:

Values of these functions to 9,10 , or 11 significant figures from 0 to 10 at intervals of 0.001 . with auxiliary tables.

(1940) XXXVII + 225 pages; bound in buckram, $\$ 2.00$.

MT\%. Table or Natural Logarithm, Volume I:

Logarithms of the integers from 1 to 50,000 to 16 places of decimals.

(1941) XVIII+ 501 pages; bound in buckram, $\$ 2.00$.

MT8. Tables of Probability Functions, Volumb I:

Values of these functions to 15 places of decimals from 0 to 1 at intervals of 0.0001 and 1 to 5.6 at intervals of 0.001 .

(1941) XXVIII+302 pages; bound in buckram, $\$ 2.00$.

MT9. Tablb of Natural Logarithms, Volumb II:

Logarithms of the integers from 50,000 to 100,000 to 16 places of decimals.

(1941) XVIII+501 pages; bound in buckram, \$2.00. 
MT10. Tablb of Natural Logartrims, Volumb III:

Logarithms of the decimal numbers from 0.0001 to 5.0000 , to 16 places of decimals. (1941) XVIII + 501 pages; bound in buckram, $\$ 2.00$.

MT11. Tables of the Mombnts of Inertia and Section Moduli of Ordinary Anglbs, Channgle, and Bulb Angles with Certatn Platb Combenations.

(1941) XIII+197 pages; bound in green cloth. \$1.25.

MT12. Table of Natural Logarithms, Volume IV:

Logarithms of the decimal numbers from 5.0000 to 10.0000 , to 16 places of decimals.

(1941) XXII + 506 pages; bound in buckram, $\$ 2.00$.

Payment is required in advance. Make remittance payable to the "National Bureau of Standards", and send with order, using the blank form facing page 3 of the cover.

The prices are for delivery in the United States and its possessions and in countries extending the franking privilege. To other countries the price of MT1 is 65 cents; that of MT2, MT3, MT4, MT5, MT6, MT7, MT8, MT9, MT10, and MT12 is $\$ 2.50$ each; and that of MT11 is $\$ 1.50$; remittance to be made payable in United States currency.

Copies of these publications have been sent to various Government depositories throughout the country, such as public libraries in large cities, and colleges and universities, where they may be consulted.

A mailing list is maintained for those who desire to receive announcements regarding new tables as they become available. A list of the tables it is planned to publish will be sent on request. 